\title{
Boundary Layers and Shock Profiles for the Broadwell Model
}

\author{
Niclas Bernhoff \\ Department of Mathematics, Karlstad University, 65188 Karlstad, Sweden \\ Correspondence should be addressed to Niclas Bernhoff; niclas.bernhoff@kau.se \\ Received 26 April 2016; Accepted 19 June 2016 \\ Academic Editor: Giovanni P. Galdi \\ Copyright (C) 2016 Niclas Bernhoff. This is an open access article distributed under the Creative Commons Attribution License, \\ which permits unrestricted use, distribution, and reproduction in any medium, provided the original work is properly cited.

\begin{abstract}
We consider the existence of nonlinear boundary layers and the typically nonlinear problem of existence of shock profiles for the Broadwell model, which is a simplified discrete velocity model for the Boltzmann equation. We find explicit expressions for the nonlinear boundary layers and the shock profiles. In spite of the few velocities used for the Broadwell model, the solutions are (at least partly) in qualitatively good agreement with the results for the discrete Boltzmann equation, that is the general discrete velocity model, and the full Boltzmann equation.
\end{abstract}

\section{Introduction}

The Boltzmann equation (BE) is a fundamental equation in kinetic theory. Half-space problems for the BE are of great importance in the study of the asymptotic behavior of the solutions of boundary value problems of the BE for small Knudsen numbers $[1,2]$ and have been extensively studied both for the full $\mathrm{BE}[3,4]$ and for the discrete Boltzmann equation (DBE) [5-8]. The half-space problems provide the boundary conditions for the fluid-dynamic-type equations and Knudsen-layer corrections to the solution of the fluiddynamic-type equations in a neighborhood of the boundary. In [8] nonlinear boundary layers for the DBE, the general discrete velocity model (DVM) was considered. Existence of weakly nonlinear boundary layers was proved. Here we exemplify the theory in [8] for a simplified model, the Broadwell model [9], where the whole machinery is actually not really needed, even if it helps out. For the nonlinear Broadwell model, we obtain explicit expressions for boundary layers near a wall moving with a constant speed. The number of conditions, on the assigned data for the outgoing particles at the boundary, needed for the existence of a unique (in a neighborhood of the assigned Maxwellian at infinity) solution of the problem is in complete agreement with the results in [8] for the DBE and [3] for the full BE. Here we also want to mention a series of papers studying initial boundary value problems for the Broadwell model using Green's functions [10-16].

We also consider the question of existence of shock profiles $[17,18]$ for the same model $[9,19]$. The shock profiles can then be seen as heteroclinic orbits connecting two singular points (Maxwellians) [20]. In [20] existence of shock profiles for the DBE in the case of weak shocks was proved. We exemplify the theory in [20] for the Broadwell model, where again the whole machinery is not really needed, even if it helps out. In this way we, in a new way, obtain similar explicit solutions, not only for weak shocks, as the ones obtained in [19] for the same problem.

The paper is organized as follows. In Section 2 we introduce the Broadwell model and find explicit expressions for the nonlinear boundary layers near a wall moving with a constant speed, and in Section 3 we find explicit expressions for the shock profiles for the Broadwell model.

\section{Nonlinear Boundary Layers for the Broadwell Model Near a Moving Wall}

In this section we study boundary layers for the nonlinear Broadwell model near a wall moving with a constant speed $b$. In [8] the nonlinear boundary layers for the DBE, the general discrete velocity model (DVM) was considered. Existence of nonlinear boundary layers was proved. Here we exemplify 
the theory in [8] in the case of a simplified model, where the whole machinery is actually not really needed, even if it helps out. The same problem was considered in [21] for a mixture model, where one of the two species was modelled by the Broadwell model.

We consider the classical Broadwell model [9] in space (with velocities $\xi_{1}=(1,0,0), \xi_{2}=(-1,0,0), \xi_{3}=(0,1,0)$, $\xi_{4}=(0,-1,0), \xi_{5}=(0,0,1)$, and $\left.\xi_{6}=(0,0,-1)\right)$

$$
\begin{aligned}
\frac{\partial \tilde{f}_{1}}{\partial t}+\frac{\partial \tilde{f}_{1}}{\partial x} & =\frac{2 \sigma}{3}\left(\tilde{f}_{3} \tilde{f}_{4}+\tilde{f}_{5} \tilde{f}_{6}-2 \tilde{f}_{1} \tilde{f}_{2}\right), \\
\frac{\partial \tilde{f}_{2}}{\partial t}-\frac{\partial \tilde{f}_{2}}{\partial x} & =\frac{2 \sigma}{3}\left(\tilde{f}_{3} \tilde{f}_{4}+\tilde{f}_{5} \tilde{f}_{6}-2 \tilde{f}_{1} \tilde{f}_{2}\right), \\
\frac{\partial \tilde{f}_{3}}{\partial t} & =-\frac{2 \sigma}{3}\left(\tilde{f}_{3} \tilde{f}_{4}-\tilde{f}_{1} \tilde{f}_{2}\right), \\
\frac{\partial \tilde{f}_{4}}{\partial t} & =-\frac{2 \sigma}{3}\left(\tilde{f}_{3} \tilde{f}_{4}-\tilde{f}_{1} \tilde{f}_{2}\right), \\
\frac{\partial \tilde{f}_{5}}{\partial t} & =-\frac{2 \sigma}{3}\left(\tilde{f}_{5} \tilde{f}_{6}-\tilde{f}_{1} \tilde{f}_{2}\right), \\
\frac{\partial \tilde{f}_{6}}{\partial t} & =-\frac{2 \sigma}{3}\left(\tilde{f}_{5} \tilde{f}_{6}-\tilde{f}_{1} \tilde{f}_{2}\right),
\end{aligned}
$$

where $\sigma$ is the mutual collision cross section. For a flow axially symmetric around the $x$-axis we can reduce system (1) to (with $f_{1}=\tilde{f}_{1}, f_{2}=\widetilde{f}_{3}=\widetilde{f}_{4}=\widetilde{f}_{5}=\widetilde{f}_{6}$, and $f_{3}=\widetilde{f}_{2}$ ) [9]

$$
\begin{aligned}
\frac{\partial f_{1}}{\partial t}+\frac{\partial f_{1}}{\partial x} & =\frac{4 \sigma}{3}\left(f_{2}^{2}-f_{1} f_{3}\right), \\
4 \frac{\partial f_{2}}{\partial t} & =-\frac{8 \sigma}{3}\left(f_{2}^{2}-f_{1} f_{3}\right), \\
\frac{\partial f_{3}}{\partial t}-\frac{\partial f_{3}}{\partial x} & =\frac{4 \sigma}{3}\left(f_{2}^{2}-f_{1} f_{3}\right) .
\end{aligned}
$$

The collision invariants are

$$
\phi=\alpha(1,1,1)+\beta(1,0,-1), \quad \alpha, \beta \in \mathbb{R},
$$

and the Maxwellians (equilibrium distributions) are

$$
\begin{aligned}
& M=s^{4}\left(a^{4}, a^{2}, 1\right), \\
& \quad \text { with } s=e^{(\alpha-\beta) / 4}>0, a=e^{\beta / 4}>0, \alpha, \beta \in \mathbb{R} .
\end{aligned}
$$

The density, momentum, and internal energy can be obtained by

$$
\begin{aligned}
\rho & =f_{1}+4 f_{2}+f_{3}, \\
\rho u & =f_{1}-f_{3}, \\
2 \rho e & =f_{1}+f_{3} .
\end{aligned}
$$

Let $b$, the speed of the wall, be a real number such that

$$
b \notin\{-1,0,1\} .
$$

We define the projections $R_{+}: \mathbb{R}^{3} \rightarrow \mathbb{R}^{n^{+}}$and $R_{-}: \mathbb{R}^{3} \rightarrow$ $\mathbb{R}^{n^{-}}, n^{-}=3-n^{+}$, by

$$
\begin{aligned}
& R_{+} h=h^{+}=\left(h_{1}, \ldots, h_{n^{+}}\right), \\
& R_{-} h=h^{-}=\left(h_{n^{+}+1}, \ldots, h_{3}\right),
\end{aligned}
$$

where

$$
n^{+}=\left\{\begin{array}{ll}
0 & \text { if } b>1 \\
1 & \text { if } 0<b<1 \\
2 & \text { if }-1<b<0 \\
3 & \text { if } b<-1,
\end{array} \quad h=\left(h_{1}, h_{2}, h_{3}\right)\right.
$$

Consider the problem

$$
\begin{aligned}
D \frac{\partial f}{\partial t}+B_{0} \frac{\partial f}{\partial x} & =Q(f, f), \quad x>b t, t>0, \\
f^{+}(b t, t) & =\widetilde{C} f^{-}(b t, t)+\widetilde{\varphi}_{0}, \\
f(x, 0) & =f_{0}(x), \\
f_{0}(x) & \longrightarrow M \quad \text { as } x \longrightarrow \infty,
\end{aligned}
$$

where $f=\left(f_{1}, f_{2}, f_{3}\right), D=(1,4,1), B_{0}=(1,0,-1), M=$ $s^{4}\left(a^{4}, a^{2}, 1\right), \widetilde{C}$ is a given $n^{+} \times n^{-}$matrix, $\widetilde{\varphi}_{0} \in \mathbb{R}^{n^{+}}$, and $Q(f, f)$ is defined by the bilinear expression:

$$
Q(f, g)=\frac{2 \sigma}{3}\left(2 f_{2} g_{2}-f_{1} g_{3}-g_{1} f_{3}\right)(1,-2,1) .
$$

After the change of variables $y=x-b t$ and the transformation

$$
f=M+M^{1 / 2} h, \quad \text { with } M=s^{4}\left(a^{4}, a^{2}, 1\right),
$$

we obtain the new system

$$
\begin{aligned}
\frac{\partial h}{\partial t}+B \frac{\partial h}{\partial y}+L h & =S(h, h), \quad y>0, t>0, \\
h^{+}(0, t) & =C h^{-}(0, t)+\varphi_{0}, \\
h(y, 0) & =h_{0}(y), \\
h_{0}(y) & \longrightarrow 0 \quad \text { as } y \longrightarrow \infty,
\end{aligned}
$$

where $h=\left(h_{1}, h_{2}, h_{3}\right), B=B_{0}-b D, C$ is an $n^{+} \times n^{-}$matrix, $\varphi_{0} \in \mathbb{R}^{n^{+}}$, and

$$
\begin{aligned}
L h & =-2 M^{-1 / 2} Q\left(M, M^{1 / 2} h\right) \\
& =\frac{4 s^{4} \sigma}{3}\left(h_{1}-2 a h_{2}+a^{2} h_{3}\right)\left(1,-2 a, a^{2}\right), \\
S(h, g) & =M^{-1 / 2} Q\left(M^{1 / 2} h, M^{1 / 2} g\right) \\
& =\frac{2 s^{2} \sigma}{3}\left(2 h_{2} g_{2}-h_{1} g_{3}-g_{1} h_{3}\right)\left(1,-2 a, a^{2}\right) .
\end{aligned}
$$


Similar initial boundary value problems have been studied in a series of papers using Green's functions (with $s^{4}=$ $1 / 6, a=1)$ for $1 / \sqrt{3}<b<1$ in [10] (with $C=\left(\begin{array}{ll}c_{1} & c_{2}\end{array}\right)$ ) and [14], for $-1<b<-1 / \sqrt{3}$ in [11], for $0<b<1 / \sqrt{3}$, with $C=0$ in $[12,13,15]$, and for diffuse boundary conditions in [16].

Here we consider the stationary nonlinear system

$$
\begin{aligned}
B \frac{d h}{d y}+L h & =S(h, h), \quad h=h(y), y>0, \\
h^{+}(0) & =C h^{-}(0)+\varphi_{0}, \\
h(y) & \longrightarrow 0 \quad \text { as } y \longrightarrow \infty .
\end{aligned}
$$

The linearized collision operator

$$
L=\frac{4 s^{4} \sigma}{3}\left(\begin{array}{ccc}
1 & -2 a & a^{2} \\
-2 a & 4 a^{2} & -2 a^{3} \\
a^{2} & -2 a^{3} & a^{4}
\end{array}\right)
$$

is symmetric and semipositive and have the null-space

$$
N(L)=\operatorname{span}\left(\left(a^{2}, a, 1\right),\left(a^{2}, 0,-1\right)\right)=\operatorname{span}\left(e_{1}, e_{2}\right),
$$

for some $e_{1}$ and $e_{2}$, such that

$$
\left\langle e_{1}, e_{2}\right\rangle_{B}=\gamma_{i} \delta_{i j}, \quad i, j=1,2 .
$$

Note also that

$$
\langle S(h, h), e\rangle=0, \quad \forall e \in N(L) .
$$

Here and below, $\langle\cdot, \cdot\rangle$ denotes the Euclidean scalar product and we denote $\langle\cdot, \cdot\rangle_{B}=\langle\cdot, B \cdot\rangle$. If $b \neq a^{2} /\left(1+a^{2}\right)$ we can choose

$$
\begin{aligned}
& e_{1}=\left(a, \frac{1}{2}, 0\right), \\
& e_{2}=\left(a^{2} b, \frac{a^{3}}{2}(1-b), a^{2}(1-b)-b\right),
\end{aligned}
$$

and then

$$
\begin{aligned}
\gamma_{1} & =a^{2}-b\left(1+a^{2}\right), \\
\gamma_{2} & =\left(a^{2}-b\left(1+a^{2}\right)\right) \\
& \cdot\left(-a^{2}+\left(1-a^{4}\right) b+\left(1+a^{2}+a^{4}\right) b^{2}\right) .
\end{aligned}
$$

We let $m^{ \pm}$denote the number of the positive and negative eigenvalues of the matrix $B^{-1} L$. The numbers $n^{ \pm}$, with $n^{+}+$ $n^{-}=3$, defined above, denote the numbers of the positive and negative eigenvalues of the matrix $B$. Moreover, we let $k^{+}, k^{-}$, and $l$ denote the number of positive, negative, and zero eigenvalues of the $2 \times 2$-matrix

$$
K=\left(\begin{array}{ll}
\left\langle y_{1}, y_{1}\right\rangle_{B} & \left\langle y_{1}, y_{2}\right\rangle_{B} \\
\left\langle y_{2}, y_{1}\right\rangle_{B} & \left\langle y_{2}, y_{2}\right\rangle_{B}
\end{array}\right),
$$

where $y_{1}=\left(a^{2}, a, 1\right)$ and $y_{2}=\left(a^{2}, 0,-1\right)$. Then $m^{ \pm}=n^{ \pm}-$ $k^{ \pm}-l[22,23]$. The eigenvalues of $K$ are

$$
\lambda_{ \pm}=\eta \pm \sqrt{\eta^{2}-4 a^{2} \kappa}
$$

where

$$
\begin{aligned}
& \eta=\left(1+a^{2}\right)\left[a^{2}-1-b\left(1+a^{2}\right)\right], \\
& \kappa=-a^{2}+\left(1-a^{4}\right) b+\left(1+a^{2}+a^{4}\right) b^{2} .
\end{aligned}
$$

We find that $\operatorname{sgn}\left(\lambda_{ \pm}\right)=\operatorname{sgn}(\eta)$ if $\kappa>0, \operatorname{sgn}\left(\lambda_{ \pm}\right)= \pm 1$ if $\kappa<$ $0, \lambda_{+}=2 \eta$, and $\lambda_{-}=0$ if $\kappa=0$, but $\kappa<0$ if $\eta=0, \operatorname{sgn}(\eta)=$ $-\operatorname{sgn}(b)$ if $\kappa>0$, and

$$
\begin{aligned}
& \kappa=0 \Longleftrightarrow \\
& b=b_{ \pm}=\frac{a^{4}-1 \pm \sqrt{1+4 a^{2}+2 a^{4}+4 a^{6}+a^{8}}}{2\left(1+a^{2}+a^{4}\right)}, \\
& \kappa>0 \quad \text { if } b>b_{+}, \text {or } b<b_{-}, \\
& \kappa<0 \quad \text { if } b_{-}<b<b_{+} .
\end{aligned}
$$

Hence, we obtain the following number of positive and negative eigenvalues for different values of $b$

\begin{tabular}{c|ccccccccc}
$b$ & -1 & $b_{-}$ & 0 & $b_{+}$ & 1 \\
\hline$n^{+}$ & 3 & 2 & 2 & 2 & 1 & 1 & 1 & 0 \\
$n^{-}$ & 0 & 1 & 1 & 1 & 2 & 2 & 2 & 3 \\
$k^{+}$ & 2 & 2 & 1 & 1 & 1 & 0 & 0 & 0 \\
$k^{-}$ & 0 & 0 & 0 & 1 & 1 & 1 & 2 & 2 \\
$l$ & 0 & 0 & 1 & 0 & 0 & 1 & 0 & 0 \\
$m^{+}$ & 1 & & 0 & 0 & 1 & 0 & 0 & 1 & 0 \\
$m^{-}$ & 0 & & 1 & 0 & 0 & 1 & 0 & 0 & 1
\end{tabular}

for

$$
b_{ \pm}=\frac{a^{4}-1 \pm \sqrt{1+4 a^{2}+2 a^{4}+4 a^{6}+a^{8}}}{2\left(1+a^{2}+a^{4}\right)} .
$$

Particularly, if $a=1$ then $b_{ \pm}= \pm 1 / \sqrt{3}$.

Explicitly, the eigenvalues of the matrix $B^{-1} L$ are 0 (of multiplicity 2) and

$$
\begin{aligned}
\lambda=\frac{4 s^{4} \sigma \kappa}{3\left(b-b^{3}\right)}, \\
\quad \text { with } \kappa=-a^{2}+\left(1-a^{4}\right) b+\left(1+a^{2}+a^{4}\right) b^{2} .
\end{aligned}
$$

For $b \neq b_{ \pm}$an eigenvector corresponding to the nonzero eigenvalue $\bar{\lambda}$ is

$$
v=\left(b+b^{2}, \frac{a}{2}\left(1-b^{2}\right), a^{2}\left(b^{2}-b\right)\right) ;
$$

that is

$$
\begin{aligned}
& B^{-1} L v=\lambda v, \\
& \langle v, e\rangle_{B}=0, \quad \forall e \in N(L) .
\end{aligned}
$$


Furthermore,

$$
B^{-1} S(v, v)=k v, \quad \text { with } k=\frac{s^{2} a^{2} \sigma}{3 b}\left(1+3 b^{2}\right) .
$$

Example 1. If $a=1$, corresponding to a nondrifting Maxwellian $M$, then we get that

$$
\begin{aligned}
& b_{ \pm}= \pm \frac{1}{\sqrt{3}}, \\
& \lambda=\frac{4 s^{4} \sigma\left(3 b^{2}-1\right)}{3\left(b-b^{3}\right)}, \\
& v=\left(b+b^{2}, \frac{1-b^{2}}{2}, b^{2}-b\right), \\
& k=\frac{s^{2} \sigma}{3 b}\left(1+3 b^{2}\right) .
\end{aligned}
$$

Note that if $\varphi_{0}=0$, then we always have the trivial solution $h=0$, and if $-1<b \leq b_{-}\left(\varphi_{0}=\left(\varphi_{01}, \varphi_{02}\right)\right.$, where both $\varphi_{01}$ and $\varphi_{02}$ must be zero, i.e., $\left.\varphi_{01}=\varphi_{02}=0\right), 0<b \leq b_{+}$ $\left(\varphi_{0} \in \mathbb{R}\right.$, where $\varphi_{0}$ must be zero, i.e., $\varphi_{0}=0$ ), or $1<b$ (no boundary conditions at all at the wall), then we have no other solutions. Otherwise, we have solutions if and only if $h_{0} \in$ $\operatorname{span}\left(v^{+}-C v^{-}\right)$.

Below we consider the remaining different cases.

(i) If $b_{+}<b<1$ then $C=\left(\begin{array}{ll}c_{1} & c_{2}\end{array}\right)$ and $\varphi_{0} \in \mathbb{R}$. Hence, if $\varphi_{0} \neq 0$ we obtain the unique solution

$$
h(y)=\frac{\lambda}{k+D e^{\lambda y}}\left(b+b^{2}, \frac{a}{2}\left(1-b^{2}\right), a^{2}\left(b^{2}-b\right)\right)
$$

with

$$
D=\frac{2\left(b+b^{2}\right)-c_{1} a\left(1-b^{2}\right)-2 c_{2} a^{2}\left(b^{2}-b\right)}{2 h_{0}} \lambda-k .
$$

(ii) If $b_{-}<b<0$ then $C=\left(\begin{array}{l}c_{1} \\ c_{2}\end{array}\right)$ and $\varphi_{0}=\left(\varphi_{01}, \varphi_{02}\right) \in \mathbb{R}^{2}$. Hence, if $\varphi_{01} \neq 0, c_{1} \neq 2 b / a(1-b)$, and

$$
\varphi_{02}=\frac{a\left(1-b^{2}\right)-2 c_{2} a^{2}\left(b^{2}-b\right)}{2\left(b+b^{2}\right)-2 c_{1} a^{2}\left(b^{2}-b\right)} \varphi_{01}
$$

then we obtain the unique solution (32) with

$$
D=\frac{b+b^{2}-c_{1}^{2} a\left(b^{2}-b\right)}{\varphi_{01}} \lambda-k
$$

and if $c_{1}=2 b / a(1-b), c_{2} \neq-(1+b) / 2 a b, \varphi_{01}=0$, and $\varphi_{02} \neq 0$, then we obtain the unique solution (32) with

$$
D=\frac{a\left(1-b^{2}\right)-2 c_{2} a^{2}\left(b^{2}-b\right)}{2 \varphi_{02}} \lambda-k .
$$

(iii) If $b<-1$ then $C=0$ and $\varphi_{0}=\left(\varphi_{01}, \varphi_{02}, \varphi_{03}\right) \in \mathbb{R}^{3}$. Hence, if $\varphi_{01} \neq 0$ and

$$
\begin{aligned}
\varphi_{02} & =\frac{a(1-b)}{2 b} \varphi_{01}, \\
\varphi_{03} & =\frac{a^{2}(b-1)}{1+b} \varphi_{01},
\end{aligned}
$$

then we obtain the unique solution (32), where

$$
D=\frac{b+b^{2}}{\varphi_{01}} \lambda-k
$$

We note that in each of the above cases $k^{+}$conditions on the assigned data $\varphi_{0}$ are implied to have a unique solution. This is in good agreement with the results for the DBE in [8] and for the continuous BE in [3].

Remark 2. Similar results can be obtained for the (reduced) plane Broadwell model

$$
\begin{aligned}
(1-b) \frac{d f_{1}}{d y} & =\sigma\left(f_{2}^{2}-f_{1} f_{3}\right), \\
-2 b \frac{d f_{2}}{d y} & =-2 \sigma\left(f_{2}^{2}-f_{1} f_{3}\right), \\
-(1+b) \frac{d f_{3}}{d y} & =\sigma\left(f_{2}^{2}-f_{1} f_{3}\right) .
\end{aligned}
$$

Particularly, with $a=1$ we have

$$
\begin{aligned}
& b_{ \pm}= \pm \frac{1}{\sqrt{2}}, \\
& \lambda=\frac{2 s^{4} \sigma\left(2 b^{2}-1\right)}{3\left(b-b^{3}\right)}, \\
& v=\left(b+b^{2}, 1-b^{2}, b^{2}-b\right), \\
& k=\frac{s^{2} \sigma}{b} .
\end{aligned}
$$

\section{Shock Profiles}

In this section we are concerned with the existence of shock profiles $[17,18]$

$$
F=F\left(x^{1}, \xi, t\right)=f\left(x^{1}-b t, \xi\right)
$$

for the Boltzmann equation

$$
\frac{\partial F}{\partial t}+\xi \cdot \nabla_{\mathbf{x}} F=Q(F, F) .
$$

Here $\mathbf{x}=\left(x^{1}, \ldots, x^{d}\right) \in \mathbb{R}^{d}, \xi=\left(\xi^{1}, \ldots, \xi^{d}\right) \in \mathbb{R}^{d}$, and $t \in \mathbb{R}_{+}$denote position, velocity, and time, respectively. Furthermore, $b$ denotes the speed of the wave. The profiles are assumed to approach two given Maxwellians

$$
M_{ \pm}=\frac{\rho_{ \pm}}{\left(2 \pi T_{ \pm}\right)^{d / 2}} e^{-\left|\xi-\mathbf{u}_{ \pm}\right|^{2} /\left(2 T_{ \pm}\right)}
$$


( $\rho, \mathbf{u}$, and $T$ denote density, bulk velocity, and temperature, resp.) as $x \rightarrow \pm \infty$, which are related through the RankineHugoniot conditions.

The (shock wave) problem is to find a solution $f=f(y, \xi)$ $\left(y=x^{1}-b t\right)$ of the equation

$$
\left(\xi^{1}-b\right) \frac{\partial f}{\partial y}=Q(f, f),
$$

such that

$$
f \longrightarrow M_{ \pm} \text {as } y \longrightarrow \pm \infty \text {. }
$$

In [20] the shock wave problem (44), (45) for the DBE was considered. Existence of shock profiles in the case of weak shocks was proved. Here we exemplify the theory in [20] in the case of a simplified model, where the whole machinery is actually not really needed, even if it helps out. In this way we, in a different way, obtain similar results as is obtained in [19] for the same problem.

We study the reduced system (2) of the classical Broadwell model in (1) [9] in space. The collision invariants are given by (3) and the Maxwellians (equilibrium distributions) by (4).

The shock wave problem for the Broadwell model reads

$$
B \frac{d f}{d y}=Q(f, f), \quad \text { where } f \longrightarrow M_{ \pm} \text {as } y \longrightarrow \pm \infty \text {, }
$$

where $B=B(b)=\operatorname{diag}(1-b,-4 b,-(1+b)), f=\left(f_{1}, f_{2}, f_{3}\right)$, and $Q(f, f)$ is defined by the bilinear expression (10).

The density $\rho$, momentum $\rho u$, and internal energy $2 \rho E$ can be obtained by (5). The Maxwellians $M_{-}=s_{-}^{4}\left(a_{-}^{4}, a_{-}^{2}, 1\right)$ and $M_{+}=s_{+}^{4}\left(a_{+}^{4}, a_{+}^{2}, 1\right)$ must fulfill the Rankine-Hugoniot conditions

$$
\begin{aligned}
\rho_{+}\left(u_{+}-b\right) & =\rho_{-}\left(u_{-}-b\right), \\
\rho_{+}\left(2 E_{+}-b u_{+}\right) & =\rho_{-}\left(2 E_{-}-b u_{-}\right),
\end{aligned}
$$

with

$$
\begin{aligned}
\rho_{ \pm} & =s_{ \pm}^{4}\left(1+4 a_{ \pm}^{2}+a_{ \pm}^{4}\right), \\
\rho_{ \pm} u_{ \pm} & =s_{ \pm}^{4}\left(a_{ \pm}^{4}-1\right), \\
2 \rho_{ \pm} E_{ \pm} & =s_{ \pm}^{4}\left(1+a_{ \pm}^{4}\right) .
\end{aligned}
$$

After some manipulations we obtain that

$$
2 E_{ \pm}=\frac{1}{3}\left(2 \sqrt{1+3 u_{ \pm}^{2}}-1\right) .
$$

We consider

$$
B \frac{d f}{d y}=Q(f, f), \quad \text { where } f \longrightarrow M_{+} \text {as } y \longrightarrow \infty
$$

and denote

$$
\begin{aligned}
& F=M+M^{1 / 2} h, \\
& \quad \text { with } M=M_{+}=s_{+}^{4}\left(a_{+}^{4}, a_{+}^{2}, 1\right)=s^{4}\left(a^{4}, a^{2}, 1\right) .
\end{aligned}
$$

Then we obtain

$$
B \frac{d h}{d y}+L h=S(h, h), \quad \text { where } h \longrightarrow 0 \text { as } y \longrightarrow \infty,
$$

with the linearized operator $L$ and the quadratic part $S(h, h)$ given by (13). The linearized collision operator is given by (15) and then fulfills properties (16)-(20).

We assume that $B$ is nonsingular; that is $b \notin\{-1,0,1\}$. Then by (52) we obtain the system

$$
\frac{d h}{d y}+B^{-1} L h=B^{-1} S(h, h) .
$$

In (25) we obtain that

$$
\begin{aligned}
b_{ \pm} & =\frac{a^{4}-1 \pm \sqrt{1+4 a^{2}+2 a^{4}+4 a^{6}+a^{8}}}{2\left(1+a^{2}+a^{4}\right)} \\
& =\frac{2 u_{+} \pm 2 \sqrt{2 E_{+}}}{1+6 E_{+}}
\end{aligned}
$$

and the eigenvalues of the matrix $B^{-1} L$ are 0 (of multiplicity 2) and

$$
\begin{aligned}
\lambda= & \frac{4 s^{4} \sigma \kappa}{3\left(b-b^{3}\right)} \\
= & \frac{\rho_{+} \sigma}{3\left(b-b^{3}\right)}\left(2 E_{+}-1-4 u_{+} b+\left(1+6 E_{+}\right) b^{2}\right), \\
& \quad \text { with } \kappa=-a^{2}+\left(1-a^{4}\right) b+\left(1+a^{2}+a^{4}\right) b^{2} .
\end{aligned}
$$

Let

$$
h=\vartheta e_{1}+\chi e_{2}+\mu v
$$

where $e_{1}$ and $e_{2}$ are eigenvectors (19) corresponding to the zero eigenvalue and $v$ is eigenvector (28) corresponding to the nonzero eigenvalue $\lambda$. Then

$$
\frac{d \vartheta}{d y}=\frac{d \chi}{d y}=0
$$

which implies that

$$
\vartheta=\chi=0,
$$

since $\lim _{y \rightarrow \infty} \vartheta=\lim _{y \rightarrow \infty} \chi=0$. Therefore

$$
\frac{d \mu}{d y}+\lambda \mu=k \mu^{2}
$$

where $k$ is given in (30). We obtain that

$$
\mu=\frac{\lambda}{k+D e^{\lambda y}} .
$$

Assume that $D \neq 0$ and let

$$
\begin{gathered}
b_{+}<b<1 \\
\text { or } b_{-}<b \leq-\frac{1}{1+2 a^{2}} .
\end{gathered}
$$


Then

$$
\begin{aligned}
& \lim _{y \rightarrow \infty} \mu=0, \\
& \lim _{y \rightarrow-\infty} \mu=\frac{\lambda}{k},
\end{aligned}
$$

and therefore

$$
h(y)=\frac{\lambda}{k+D e^{\lambda y}} v, \quad D \neq 0
$$

We conclude that the solution of system (50) is of the form

$$
f(y)=M_{+}+\frac{\lambda}{k+D e^{\lambda y}} M_{+}^{1 / 2} v
$$

where $\lambda=\frac{4 s^{4} \sigma}{3\left(b-b^{3}\right)}\left(-a^{2}+\left(1-a^{4}\right) b+\left(1+a^{2}+a^{4}\right) b^{2}\right), k=\frac{s^{2} a^{2} \sigma}{3 b}\left(1+3 b^{2}\right), v=\left(b+b^{2}, \frac{a}{2}\left(1-b^{2}\right), a^{2}\left(b^{2}-b\right)\right), D \neq 0$.

It follows that

$$
\begin{aligned}
M_{-}= & M_{+}+\frac{\lambda}{k} M_{+}^{1 / 2} v \\
= & \frac{s^{4}}{1+3 b^{2}}\left[p^{2} \frac{1+b}{1-b}, p q, q^{2} \frac{1-b}{1+b}\right], \\
& \text { with } p=\left(2+a^{2}\right) b-a^{2}, q=1+\left(1+2 a^{2}\right) b,
\end{aligned}
$$

which is a Maxwellian. Formally we can allow $b<-1$ and $-1 /\left(1+2 a^{2}\right)<b<0$. However, then, the equilibrium distribution (65) will not be nonnegative and, hence, not a Maxwellian.

We note that

$$
\begin{aligned}
& f(y)=\Theta(y) M_{+}+(1-\Theta(y)) M_{-}, \\
& \text {with } \Theta(y)=\frac{1}{1+C e^{-\lambda y}},
\end{aligned}
$$

where $C=k / D \neq 0$ is an arbitrary nonzero constant. The structure coincides with the one for the Mott-Smith approximation [24] in [25]. However, $\lambda$ is obtained in different ways.

Remark 3. We can instead of system (50) consider

$$
B \frac{d f}{d y}=Q(f, f), \quad \text { where } f \longrightarrow M_{-} \text {as } y \longrightarrow-\infty
$$

with

$$
\begin{aligned}
& \frac{a^{2}}{2+a^{2}}<b<b_{+}, \\
& \text {or }-1<b<b_{-},
\end{aligned}
$$

and in a similar way as above, we obtain

$$
\begin{aligned}
f(y) & =M_{-}+\frac{\lambda}{k+D e^{\lambda y}} M_{-}^{1 / 2} v, \\
M_{+} & =M_{-}+\frac{\lambda}{k} M_{-}^{1 / 2} v .
\end{aligned}
$$

Example 4. If $a=1$ then we have

$$
\begin{aligned}
\rho_{+} & =6 s_{+}^{4}, \\
u_{+} & =0, \\
E_{+} & =\frac{1}{6}, \\
b_{ \pm} & = \pm \frac{1}{\sqrt{3}}, \\
\lambda & =\frac{4 s^{4} \sigma\left(3 b^{2}-1\right)}{3\left(b-b^{3}\right)}, \\
v & =\left(b+b^{2}, \frac{1-b^{2}}{2}, b^{2}-b\right), \\
k & =\frac{s^{2} \sigma}{3 b}\left(1+3 b^{2}\right) .
\end{aligned}
$$

Furthermore,

$$
\begin{aligned}
& f(y)=M_{+}+\frac{\lambda}{k+D e^{\lambda y}} M_{+}^{1 / 2} v=s^{4}(1,1,1) \\
& +\frac{4 s^{4}\left(3 b^{2}-1\right)}{\left(1+3 b^{2}\right)+\widetilde{D} e^{\lambda y}}\left(\frac{b}{1-b}, \frac{1}{2}, \frac{-b}{1+b}\right) \\
& \quad=r\left[\left((3 b-1)^{2} \frac{1+b}{1-b},\left(9 b^{2}-1\right),(3 b+1)^{2} \frac{1-b}{1+b}\right)\right. \\
& \left.+\widetilde{D} e^{\lambda y}(1,1,1)\right], \quad \text { where } r=\frac{s^{4}}{\left(1+3 b^{2}\right)+\widetilde{D} e^{\lambda y}},
\end{aligned}
$$

and the other Maxwellian is

$$
\begin{gathered}
M_{-}=\frac{s^{4}}{1+3 b^{2}}\left((3 b-1)^{2} \frac{1+b}{1-b},(3 b+1)\right. \\
\left.\cdot(3 b-1),(3 b+1)^{2} \frac{1-b}{1+b}\right) .
\end{gathered}
$$


Example 5. Similar results can be obtained for the (reduced) plane Broadwell model

$$
\begin{aligned}
(1-b) \frac{d f_{1}}{d y} & =\sigma\left(f_{2}^{2}-f_{1} f_{3}\right), \\
-2 b \frac{d f_{2}}{d y} & =-2 \sigma\left(f_{2}^{2}-f_{1} f_{3}\right), \\
-(1+b) \frac{d f_{3}}{d y} & =\sigma\left(f_{2}^{2}-f_{1} f_{3}\right) .
\end{aligned}
$$

Particularly, with $a=1$ we have

$$
\begin{aligned}
\rho_{+} & =4 s_{+}^{4}, \\
u_{+} & =0, \\
E_{+} & =\frac{1}{4}, \\
c_{ \pm} & = \pm \frac{1}{\sqrt{2}}, \\
\lambda & =\frac{2 s^{4} \sigma\left(2 b^{2}-1\right)}{3\left(b-b^{3}\right)}, \\
v & =\left(b+b^{2}, 1-b^{2}, b^{2}-b\right), \\
k & =\frac{s^{2} \sigma}{b} .
\end{aligned}
$$

The other Maxwellian is then

$$
\begin{aligned}
M_{-} & =s^{4}\left((2 b-1)^{2} \frac{1+b}{1-b},(2 b+1)(2 b-1),(2 b+1)^{2}\right. \\
& \left.\cdot \frac{1-b}{1+b}\right) .
\end{aligned}
$$

The shock strength (cf. [19]) is given by the density ratio

$$
\begin{aligned}
\sigma_{\rho} & =\frac{\rho_{-}-\rho_{+}}{\rho_{+}}=\frac{2 a^{2}}{\rho_{+}} \frac{\lambda}{k} \\
& =\frac{8 s^{2}}{\rho_{+}} \frac{b+b^{2}+a^{2}\left(b^{2}-1\right)+a^{4}\left(b^{2}-b\right)}{\left(1-b^{2}\right)\left(1+3 b^{2}\right)}
\end{aligned}
$$

if $b>0$ and if $b<0$ by

$$
\begin{aligned}
\sigma_{\rho} & =\frac{\rho_{-}-\rho_{+}}{\rho_{-}}=\frac{2 a^{2}}{\rho_{-}} \frac{\lambda}{k} \\
& =\frac{8 s^{2}}{\rho_{-}} \frac{b+b^{2}+a^{2}\left(b^{2}-1\right)+a^{4}\left(b^{2}-b\right)}{\left(1-b^{2}\right)\left(1+3 b^{2}\right)} .
\end{aligned}
$$

Then the shock strength $\sigma_{\rho}$ tends to infinity as $b$ approaches 1 and to zero as $b$ approaches $b_{ \pm}$; that is

$$
\begin{gathered}
\sigma_{\rho} \longrightarrow \infty \quad \text { as } b \longrightarrow 1 \\
\sigma_{\rho} \longrightarrow 0 \quad \text { as } b \longrightarrow b_{ \pm} .
\end{gathered}
$$

The shock width (cf. [19]) is given by the density ratio

$$
\begin{aligned}
d_{\rho} & =\frac{\left|\rho_{-}-\rho_{+}\right|}{\max _{y}|d \rho / d y|}=\frac{4}{\lambda} \\
& =\frac{3 b\left(1-b^{2}\right)}{s^{4} \sigma\left(b+b^{2}+a^{2}(b-1)+a^{4}\left(b^{2}-b\right)\right)}
\end{aligned}
$$

or by the velocity ratio

$$
d_{u}=\frac{\left|u_{-}-u_{+}\right|}{\max _{y}|d u / d y|}=\frac{4}{\lambda} \frac{\left|1-u_{-} / b\right|}{\rho_{+}} .
$$

We conclude that the shock widths $d_{\rho}$ and $d_{u}$ tend to zero as $b$ approaches 1 and to infinity as $b$ approaches $b_{ \pm}$; that is

$$
\begin{aligned}
& d_{\rho} \longrightarrow 0, \\
& d_{u} \longrightarrow 0 \\
& \quad \text { as } b \longrightarrow 1, \\
& d_{\rho} \longrightarrow \infty, \\
& d_{u} \longrightarrow \infty \quad \text { as } b \longrightarrow b_{ \pm} .
\end{aligned}
$$

\section{Competing Interests}

The author declares that there is no conflict of interests regarding the publication of this paper.

\section{References}

[1] Y. Sone, Kinetic Theory and Fluid Dynamics, Modeling and Simulation in Science, Engineering and Technology, Birkhäuser, 2002.

[2] Y. Sone, Molecular Gas Dynamics, Birkhäuser, Boston, Mass, USA, 2007.

[3] S. Ukai, T. Yang, and S.-H. Yu, "Nonlinear boundary layers of the Boltzmann equation: I. Existence," Communications in Mathematical Physics, vol. 236, no. 3, pp. 373-393, 2003.

[4] C. Bardos, F. Golse, and Y. Sone, "Half-space problems for the Boltzmann equation: a survey," Journal of Statistical Physics, vol. 124, no. 2-4, pp. 275-300, 2006.

[5] S. Ukai, "On the half-space problem for the discrete velocity model of the Boltzmann equation," in Advances in Nonlinear Partial Differential Equations and Stochastics, S. Kawashima and T. Yanagisawa, Eds., pp. 160-174, World Scientific, 1998.

[6] S. Kawashima and S. Nishibata, "Existence of a stationary wave for the discrete Boltzmann equation in the half space," Communications in Mathematical Physics, vol. 207, no. 2, pp. 385-409, 1999.

[7] S. Kawashima and S. Nishibata, "Stationary waves for the discrete Boltzmann equation in the half space with reflective boundaries," Communications in Mathematical Physics, vol. 211, no. 1, pp. 183-206, 2000.

[8] N. Bernhoff, "On half-space problems for the weakly non-linear discrete Boltzmann equation," Kinetic and Related Models, vol. 3, no. 2, pp. 195-222, 2010. 
[9] J. E. Broadwell, "Shock structure in a simple discrete velocity gas," Physics of Fluids, vol. 7, no. 8, pp. 1243-1247, 1964.

[10] C.-Y. Lan, H.-E. Lin, and S.-H. Yu, "The Green's functions for the Broadwell model in a half space problem," Networks and Heterogeneous Media, vol. 1, no. 1, pp. 167-183, 2006.

[11] S. Deng, W. Wang, and S.-H. Yu, "Pointwise convergence to a Maxwellian for a Broadwell model with a supersonic boundary," Networks and Heterogeneous Media, vol. 2, no. 3, pp. 383-395, 2007.

[12] S. Deng, W. Wang, and S.-H. Yu, "Pointwise convergence to Knudsen layers of the Boltzmann equation," Communications in Mathematical Physics, vol. 281, no. 2, pp. 287-347, 2008.

[13] C.-Y. Lan, H.-E. Lin, and S.-H. Yu, “The Green's function for the Broadwell model with a transonic boundary," Journal of Hyperbolic Differential Equations, vol. 5, no. 2, pp. 279-294, 2008.

[14] S. Deng, W. Wang, and S.-H. Yu, "Broadwell model and conservative supersonic boundary," Archive for Rational Mechanics and Analysis, vol. 200, no. 1, pp. 203-223, 2011.

[15] H.-E. Lin, "Nonlinear stability of the initial-boundary value problem for the Broadwell model around a Maxwellian," Journal of Hyperbolic Differential Equations, vol. 8, no. 1, pp. 131-157, 2011.

[16] S. Deng, W. Wang, and S.-H. Yu, "Bifurcation on boundary data for linear Broadwell model with conservative boundary condition," Journal of Hyperbolic Differential Equations, vol. 11, no. 3, pp. 603-619, 2014.

[17] R. E. Caflisch and B. Nicolaenko, "Shock profile solutions of the Boltzmann equation," Communications in Mathematical Physics, vol. 86, no. 2, pp. 161-194, 1982.

[18] T.-P. Liu and S.-H. Yu, "Boltzmann equation: micro-macro decompositions and positivity of shock profiles," Communications in Mathematical Physics, vol. 246, no. 1, pp. 133-179, 2004.

[19] R. E. Caflisch, "Navier-Stokes and Boltzmann shock profiles for a model of gas dynamics," Communications on Pure and Applied Mathematics, vol. 32, no. 4, pp. 521-554, 1979.

[20] N. Bernhoff and A. Bobylev, "Weak shock waves for the general discrete velocity model of the Boltzmann equation," Communications in Mathematical Sciences, vol. 5, no. 4, pp. 815-832, 2007.

[21] N. Bernhoff, "Boundary layers and shock profiles for the discrete Boltzmann equation for mixtures," Kinetic and Related Models, vol. 5, no. 1, pp. 1-19, 2012.

[22] A. V. Bobylev and N. Bernhoff, "Discrete velocity models and dynamical systems," in Lecture Notes on the Discretization of the Boltzmann Equation, N. Bellomo and R. Gatignol, Eds., vol. 63 of Series on Advances in Mathematics for Applied Sciences, pp. 203-222, World Scientific, 2003.

[23] N. Bernhoff, "On half-space problems for the linearized discrete Boltzmann equation," Rivista di Matematica della Università di Parma. Serie 7, vol. 9, pp. 73-124, 2008.

[24] H. M. Mott-Smith, "The solution of the Boltzmann equation for a shock wave," Physical Review, vol. 82, pp. 885-892, 1951.

[25] A. V. Bobylev, M. Bisi, M. P. Cassinari, and G. Spiga, "Shock wave structure for generalized Burnett equations," Physics of Fluids, vol. 23, no. 3, Article ID 030607, 2011. 


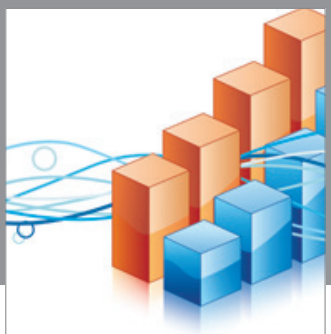

Advances in

Operations Research

vatem alat4

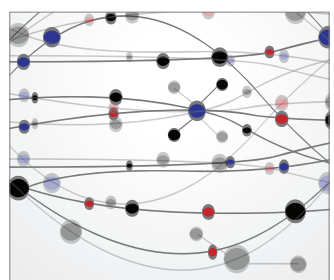

\section{The Scientific} World Journal
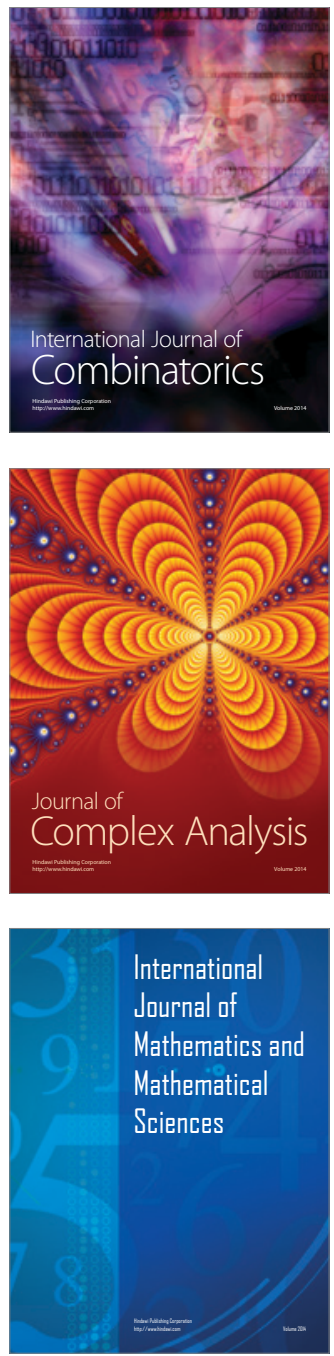
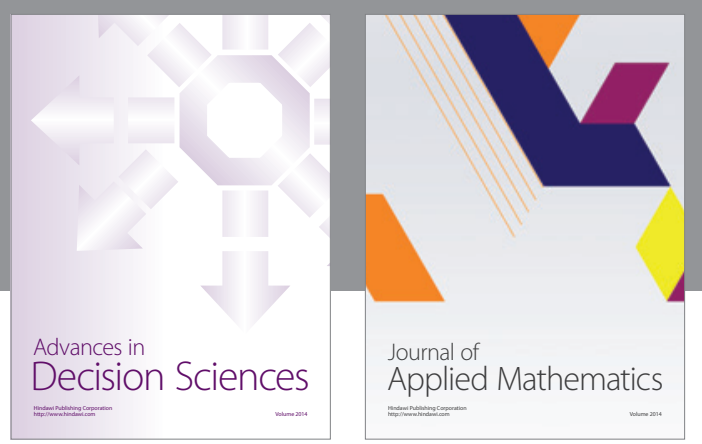

Algebra

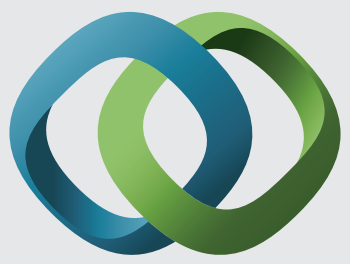

\section{Hindawi}

Submit your manuscripts at

http://www.hindawi.com
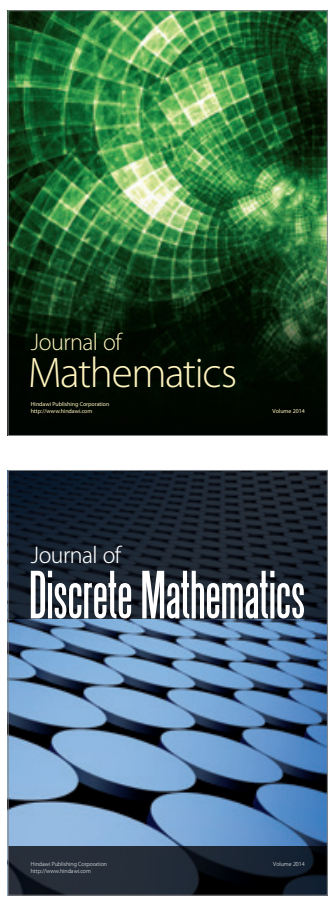

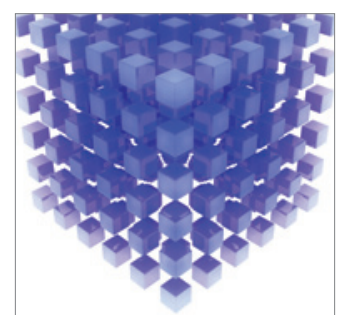

Mathematical Problems in Engineering
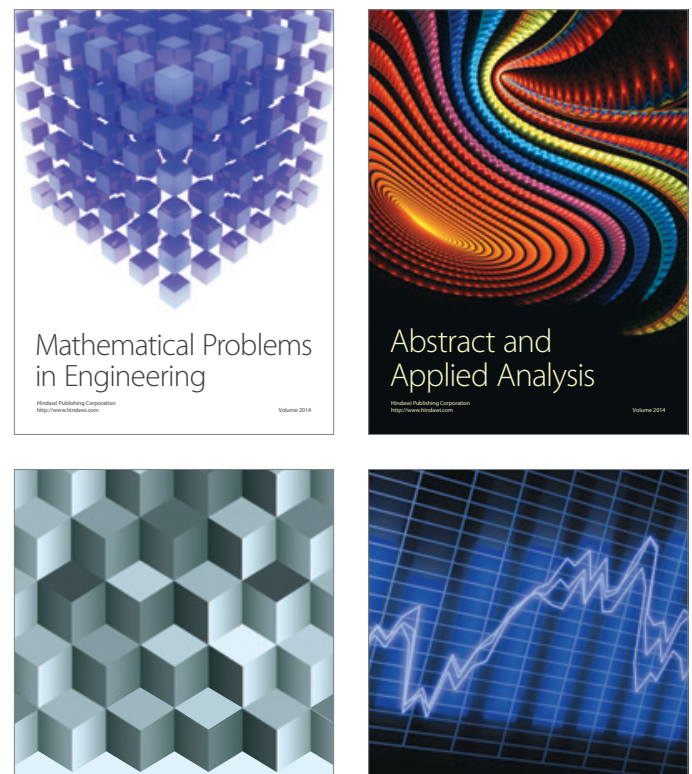

Journal of

Function Spaces

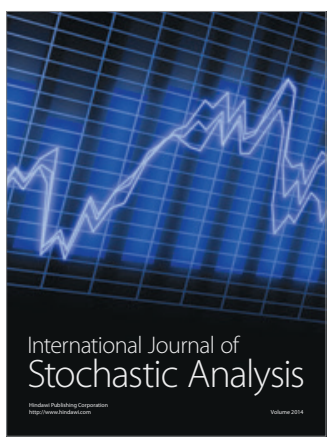

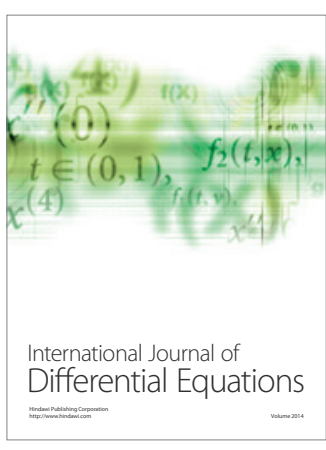
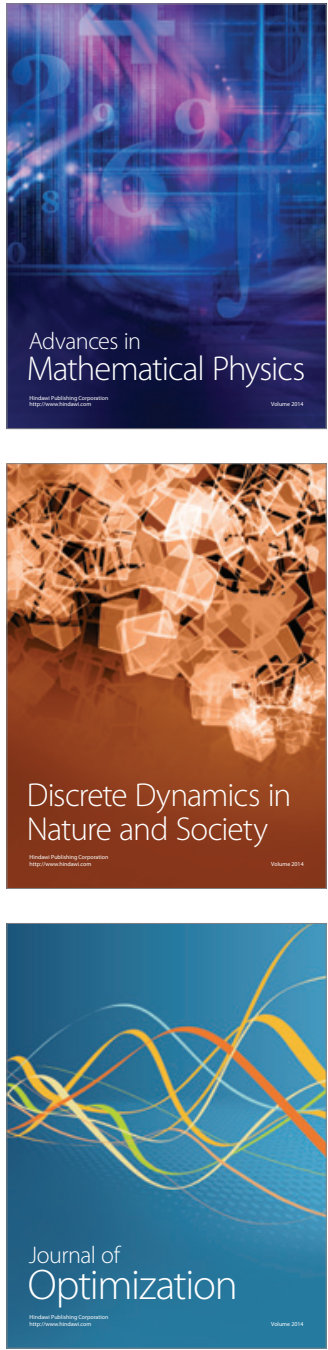